\title{
Planejamento de programas de reciclagem de resíduos de CONSTRUÇÃO E DEMOLIÇÃO NO BRASIL: UMA ANÁLISE MULTIVARIADA
}

\author{
PlanNing of CONSTRUCtion AND demolition WASTE ReCYCLING \\ PROGRAMS IN BRAZIL: A MULTIVARIATE ANALYSIS
}

\author{
NÉBEL DA COSTA \\ Engenheira Industrial (UNI, Nicarágua). Doutora em Engenharia de Produção (UFSC) \\ NEWTON DA COSTA JR. \\ Doutor em Administração (EAESP/FGV). Professor do Departamento de Economia da UFSC
}

\begin{abstract}
MÔNICA LUNA
Doutor em Engenharia de Produção (UFSC). Professora do Departamento de Engenharia Mecânica e de Produção da UFC
\end{abstract}

\section{Paulo Selig}

Doutor em Engenharia de Produção (UFSC). Professor do Departamento de Engenharia de Produção da UFSC

$$
\text { JANAÍDE ROCHA }
$$

Doutor em Engenharia Civil (INSA, França). Professora do Departamento de Engenharia Civil da UFSC

\author{
Recebido: 31/07/06 Aceito: 12/11/07
}

\begin{abstract}
RESUMO
Este trabalho desenvolve um modelo, baseado em técnicas exploratórias de dados e de estatística multivariada, que permite identificar as variáveis mais relevantes associadas ao sucesso da implementação de programas de reciclagem de resíduos de construção e demolição (RCD) nos municípios brasileiros. Os resultados da pesquisa mostraram que existem diferenças marcantes entre as cidades que implantaram programas de reciclagem de RCD e aquelas que não implantaram. As variáveis mais significativas encontradas foram: percentual de funcionários de nível médio que trabalham na prefeitura, renda média anual do município, percentual de domicílios com água, existência de programas de coleta seletiva de lixo, programas de incentivo para geraçáo de trabalho e renda e existência de áreas de recepção de entulhos, a maioria relacionadas à gestão municipal.
\end{abstract}

PALAVRAS-CHAVE: Resíduo de construção e demolição, reciclagem, análise multivariada.

\begin{abstract}
This work develops a model, based on exploratory data analysis and multivariate statistics, to identify the regional variables that most contribute to the success in the implementation of construction and demolition waste (CDW) recycling programs in Brazilian cities. The results showed noteworthy differences between cities that had implemented programs of $C D W$ recycling and those that did not. The most significant variables were the number of mid-level education employees in the city hall, the city annual average income, the percentage of household water supply connections, the existence of programs of selective domestic waste collection, programs to stimulate work and income and areas of waste deposition. Most of the variables are related to city administration.
\end{abstract}

KEYWORDS: Construction and demolition waste, recycling, multivariate analysis.

\section{INTRODUÇÃO}

Dentro da problemática da poluição ambiental, um fator importante refere-se ao aumento da geração dos resíduos de construção e demolição (RCD). Estes resíduos, segundo Chung e Lo (2003), representam, aproximadamente, de 20 a $30 \%$ do fluxo de resíduos sólidos gerados pelas cidades dos países desenvolvidos, sendo que muitas vezes este valor pode chegar a mais de 50\% do total de resíduos sólidos produzidos.

Nas cidades brasileiras de médio e grande porte, segundo Pinto (1999), os resíduos provenientes de construções e demoliçôes representam de 40 a $70 \%$ da massa total dos resíduos sólidos urbanos. Consequentemente, este tipo de resíduo tem recebido grande atenção por parte de prefeituras e de órgãos responsáveis devido aos problemas causados pela sua deposição indevida, pois aquelas têm que arcar com os custos de sua remoção quando os infratores não são identificados. 
Diante deste panorama e considerando que por volta de 88 a $95 \%$ destes resíduos são de interesse para a reciclagem como agregados para a construção civil (Sardá, 2003; Angulo et al, 2002), foi lançada a resolução $\mathrm{N}^{\circ} 307$ do CONAMA, em julho de 2002, que obriga os geradores e prefeituras a tomarem medidas para a diminuição, a reciclagem e a disposição adequada dos resíduos de construção e demolição gerados.

Para Languell (2001), o sucesso de um programa de desconstrução, que resulta no reuso e na reciclagem de materiais, depende muito de fatores regionais, e estes fatores devem ser avaliados para medir se uma determinada cidade ou região apresenta condições favoráveis ou não ao sucesso da implantação.

Portanto, estudos que abordem a gestão dos resíduos e a sua reciclagem considerando seus aspectos macroambientais são cada vez mais relevantes. Estes estudos podem servir para apoiar municipalidades, tomadores de decisão e empresários na elaboração de planos e programas de gerenciamento de resíduos e reciclagem que estejam mais voltados à realidade local, considerando suas características regionais.

Dentro deste contexto, o presente trabalho tem como objetivo de desenvolver um modelo quantitativo baseado em técnicas exploratórias de dados e de estatística multivariada que permita identificar e caracterizar as variáveis mais relevantes - a nível social, econômico, político-legal, gestão municipal e de aspectos técnicos da reciclagem - associadas ao sucesso da implantação de programas de reciclagem de RCD nos municípios brasileiros.

\section{A RECICLAGEM DOS RCD E SEUS FATORES DE SUCESSO}

\section{Caraterização dos resíduos de construção e demolição (RCD)}

Os resíduos de construção e demolição (RCD) são caracterizados, segundo o relatório elaborado pela Franklin Associates (1998) como: “O resíduo material que é produzido no processo de construção, renovação ou demolição de estruturas. As estruturas representam construçôes de todos os tipos (residencial e não residencial), bem como estradas e pontes".
Estes resíduos são compostos por restos de argamassa, tijolo, alvenaria, concreto, cerâmica, gesso, madeira, metais, etc. São, em sua maioria, considerados inertes e, por conseguinte, pouca atenção tem sido dada às suas práticas de gerenciamento. Além do mais, estudos indicam que os RCD apresentam em sua composição materiais perigosos ou potencialmente tóxicos para a saúde e o ecossistema (ICF, 1995). A reciclagem de RCD como estratégia de gerenciamento, enfatizada na Agenda 21, traz benefícios econômicos e ambientais para as cidades onde é implantada. Além da diminuição dos custos de gerenciamento do resíduo, o custo do produto reciclado é bem menor que o agregado natural. Brito Filho (apud Leite, 2001), observa que se obtém uma economia de $67 \%$ em média quando comparados os preços de agregado reciclado e agregado natural.

\section{Fatores regionais que afetam a reciclagem de RCD}

Os fatores que influenciam os programas de reciclagem podem ser agrupados, conforme as categorias de análise utilizadas por Wright et al (1998), para o estudo da análise do macroambiente de negócios, em: sociais, econômicos, político-legais e técnico-gerenciais. Existem outras classificações possíveis, como a proposta no documento IBGE (2002). No entanto, a adoção de uma outra classificação não alteraria os resultados, desde que as variáveis básicas dentro de cada categoria sejam as mesmas.

\section{- Aspectos sociais}

Os aspectos sociais referem-se ao crescimento populacional, educação, densidade populacional, consciência ambiental, fatores que influenciam as necessidades de infra-estrutura, geração de resíduos, atitude da sociedade para a preservação do meio ambiente e apoio $\mathrm{a}$ açôes voltadas à reciclagem. Lauritzen e Hansen (1997), ao descreverem os fatores que devem ser considerados quando avaliada a probabilidade de sucesso de um programa de reciclagem de RCD em um dado local, consideram como os mais importantes a densidade populacional, os depósitos de matériaprima virgem (agregados) e o nível de industrialização.

\section{- Aspectos econômicos}

Do ponto de vista da geração do resíduo e do apoio à reciclagem, a economia de uma região e seu crescimento se mostram como fatores importantes a serem considerados. Regiōes que apresentam economia saudável e crescimento econômico precisam de infraestrutura para apoiar esse crescimento. Normalmente sua população tem poder aquisitivo mais alto e existe oferta de emprego e bem-estar. Em sua maioria, essas economias recebem maior influxo de pessoas em busca de oportunidades de emprego e melhor qualidade de vida (Languell, 2001). Dentre os aspectos econômicos, a autora ressalta que as variáveis renda familiar, taxa de desemprego e PIB são indicadores diretamente relacionados à economia da região e de seus habitantes.

\section{- Aspectos político-legais}

Variáveis como legislação ambiental, incentivos econômicos e fiscalização estão entre as mais mencionadas, entre os aspectos político-legais, por vários autores como sendo importantes para a implementação e o sucesso da reciclagem e da desconstrução (Languell, 2001; Barth, 1994; Vincke e Rousseau, 1994; Chung e Lo, 2003). Segundo Languell (2001), depois de considerações monetárias, as regulamentações, os mandatos e as leis são as variáveis que mais influenciam os negócios de reciclagem e desconstrução. Sem uma pressão legal ou econômica para reduzir, reusar e reciclar, a maioria dos esforços é ignorada.

\section{- Aspectos técnicos e de gestão}

Aqui são agrupados vários aspectos que estão intimamente relacionados, ressaltando que todos eles estão voltados às características regionais. Os aspectos de gestão se referem às questôes que envolvem entidades governamentais, como prefeituras, e outros órgãos relacionados à gestão de resíduos, como o CONAMA (Conselho Nacional do Meio Ambiente), SINDUSCONs (Sindicato da Indústria de Construção Civil de cada estado), etc. Os aspectos técnicos se referem à quantidade de resíduo produzido, estoque de edifícios, distâncias de matéria-prima virgem, comercialização do resíduo, número de aterros, entre outros. 


\section{POPULACÃo E AMOSTRA}

A população-alvo da pesquisa foi constituída por todos os municípios brasileiros presentes no censo de 2000 e encontrados na página do IBGE, em julho de 2003. A partir desta população-alvo, selecionou-se uma amostra formada por municípios com mais de 300 mil habitantes, perfazendo um total de 64 municípios.

O motivo do procedimento acima foi devido a que a maioria dos municípios que já implementaram um sistema de reciclagem de RCD é de médio e grande porte. Do total das 64 cidades selecionadas, observou-se que 52 não implantaram programas de reciclagem de RCD (grupo 1), e 12 que os implementaram (grupo 2). Como o método multivariado utilizado proporciona melhores resultados, do ponto de vista estatístico, quando os grupos são de mesmo tamanho, ou de tamanhos não muito díspares, optou-se por diminuir a amostra de cidades sem reciclagem, conforme sugerido por Hair et al (1998). Esta diminuição foi realizada através de um procedimento de sorteio aleatório. Assim, o maior e o menor grupo ficaram constituídos por $36 \mathrm{e}$ 12 municípios, respectivamente. A Tabela 1 apresenta a composição final de cada grupo de cidades.

Como este trabalho tem natureza exploratória, foram selecionadas 28 variáveis explicativas para caracterizar as cidades. Estas foram escolhidas em função de sua disponibilidade nas páginas do IBGE e de outras fontes disponíveis na Internet, como BNDES, CEMPRE (Centro Empresarial de Reciclagem) e, principalmente, em função de serem consideradas importantes para o estudo da reciclagem em uma série de trabalhos acadêmicos e técnicos, tais como em Langell (2001), Lauritzen e Hansen (1997), entre outros.

Como algumas variáveis foram eliminadas pela falta de diversas observaçôes, a amostra final constituiu-se de 20 variáveis, que são descritas na Tabela 2 .

\section{MÉTODO, RESULTADOS E DISCUSSÃO}

As técnicas multivariadas utilizadas foram a análise discriminante e a regressão logística. Sendo que esta última foi apenas utilizada para validar os resultados da primeira.
A análise discriminante tem como objetivo estimar uma função linear de duas ou mais variáveis independentes, chamada de função discriminante, que separar ou discriminar dois ou mais grupos de observaçóes ou casos previamente definidos - no caso, cidades com e sem reciclagem de RCD. Ela é normalmente empregada para descobrir as características que distinguem os membros de um grupo dos de outro, de modo que, conhecidas as características de um novo indivíduo, se possa prever a que grupo pertence. Detalhes sobre o tema podem ser encontrados em Hair et al (1998), Johnson e Wichern (1998) e Klecka (1980), entre outros.

A seguir, passa-se a descrever as principais etapas realizadas para a estimação do modelo de análise discriminante aplicado ao planejamento e implantação de programas de reciclagem de RCD, bem como alguns resultados do uso alternativo da regressão logística.

\section{- Estimação da função discriminante}

Antes de proceder à determinação da função discriminante é necessário verificar se as variáveis de entrada obedecem a uma série de restriçôes, tais possa, da melhor maneira possível,

como: distribuição normal multivariada, matriz de variância-covariância homogênea para cada grupo (variabilidade igual dentro de cada grupo), não haver multicolinearidade entre as variáveis, entre outros aspectos.

Através de testes estatísticos verificou-se que algumas destas restriçōes não foram obedecidas. Para melhorar este problema, duas variáveis foram eliminadas devido a problemas de multicolinearidade ("domicílios com serviço de esgoto" e "empresas de construção") e usou-se uma transformação logarítmica para a variável "densidade" melhor aderir à curva normal.

Apesar dos procedimentos acima, nove das 18 variáveis não apresentaram aderência à curva normal e não se conseguiu homogeneidade entre as matrizes de variância-covariância.

No entanto, segundo Klecka (1980, p. 61) e Hair et al (1998), a técnica é razoavelmente robusta, ou seja, mesmo que algumas restrições não sejam rigorosamente obedecidas os resultados ainda podem ser perfeitamente válidos. No caso da análise discriminante, o sucesso do modelo, em última instância, pode ser medido a posteriori pela capacidade da função discriminante estimada em separar os grupos corretamente.

Tabela I - Amostra das cidades classificadas nos grupos I e 2

\begin{tabular}{ccc}
\hline \multicolumn{2}{c}{ Grupo 1 - Cidades sem reciclagem } & Grupo 2 - Cidades com \\
de RCD & Mogi das Cruzes/SP & Belo Horizonte/MG de RCD \\
\hline Aracaju/SE & Montes Claros/MG & Brasília/DF \\
Bauru/SP & Natal/RN & Campinas/SP \\
Belém/PA & Niterói/RJ & Guarulhos/SP \\
Betim/MG & Osasco/SP & Londrina/PR \\
Boa Vista/RR & Palmas/TO & Piracicaba/SP \\
Campina Grande/PB & Pelotas/RS & Ribeirão Preto/SP \\
Canoas/RS & Petrópolis/RJ & Rio de Janeiro/RJ \\
Contagem/MG & Porto Alegre/RS & São José dos Campos/SP \\
Cuiabá/MT & Porto Velho/RO & São José do Rio Preto/SP \\
Feira de Santana/BA & Recife/PE & Salvador/BA \\
Florianópolis/SC & Rio Branco/AC & São Paulo/SP \\
Fortaleza/CE & Sernardo do Campo/SP & \\
Juiz de Fora/MG & Santo André/SP & \\
Macapá/AP & Santos/SP & \\
Maceió/AL & Sorocaba/SP & \\
Manaus/AM & Teresina/PI & \\
Maringá/PR & Uberlândia/MG & \\
Mauá/SP &
\end{tabular}

Fonte: Censo 2000, IBGE

Nota: Cidades pertencentes ao grupo 2 são as que possuem unidades de reciclagem implantadas, mesmo que ainda não estejam em pleno funcionamento. 
Tabela 2 - Variáveis explicativas e sua definição

\begin{tabular}{c}
\hline Variável \\
\hline Área de recepção de entulho \\
Densidade populacional \\
Descentralização \\
administrativa (Conselhos) \\
Domicílios com água \\
Domicílios c/ coleta lixo \\
Domicílios com esgoto \\
Educação \\
Empresas de construção/ \\
empresas totais \\
Funcionários prefeitura \\
nível auxiliar \\
Funcionários prefeitura \\
nível médio \\
Funcionários prefeitura \\
nível superior \\
Habitantes/domicílio \\
Idade da cidade \\
Instrumentos de \\
legislação municipal \\
(Legislação)
\end{tabular}

Número de consórcios

Número de empresas/mil habitantes

Programa de coleta seletiva

Programas de geração de trabalho e renda (Incentivos)

Renda média anual

Taxa de crescimento populacional
Definição

Locais destinados oficialmente à deposição de entulho, visando a sua posterior reciclagem

Número de habitantes dividido pela área total do município

Meios que a administração tem para delegar atividades da gestão municipal. Foram considerados aqueles relacionados ao meio ambiente e à política urbana: conselho paritário do meio ambiente, fundo municipal do meio ambiente e conselho na área de política urbana Variável categórica com valores de zero a três

Percentual dos municípios atendidos

Percentual dos municípios atendidos

Percentual dos municípios atendidos

Percentual de analfabetos com mais de 15 anos

Índice que representa o total de empresas de construção dividido pelo total de empresas presentes no município

Percentual do número de funcionários de nível auxiliar em relação ao total de funcionários da prefeitura

Percentual do número de funcionários de nível médio em relação ao total de funcionários da prefeitura

Percentual do número de funcionários de nível superior em relação ao total de funcionários da prefeitura

Número de pessoas habitando o mesmo domicílio

Número de anos desde a fundação da cidade

Instrumentos voltados à administração do município: plano diretor, lei de zoneamento, lei orgânica do município, lei de parcelamento, código de obras e código de postura. Os elementos que compóem essa variável foram agrupados e somados, considerando sua existência ou não, sendo uma variável categórica

Representa a soma dos programas de consórcios existentes no município

Número de empresas com CNPJ reportados no censo dividido pelo número de habitantes $\mathrm{x}$ (mil)

Existência ou não desse programa no município

Mostra as condições que o município oferece para novos investimentos e criação de trabalho e renda. São eles: incentivo para atração de atividade econômica, fornecimento de infraestrutura, programa de geração de trabalho e renda e programa ou ação de capacitação profissional. Em geral, espera-se que quanto menos desenvolvido for o município, mais incentivos deve proporcionar para atrair investimentos, renda e trabalho PIB do município dividido pelo número de habitantes

Percentual do aumento anual da população num determinado período de tempo, encontrado no censo 2000
Uma vez estruturadas as variáveis e verificadas suas propriedades estatísticas, passa-se à estimação da função discriminante que é utilizada para o cálculo do escore discriminante de cada cidade. As variáveis que compóem a função discriminante não padronizada foram estimadas pelo método stepwise. Elas são apresentadas na Tabela 3 junto com o valor do teste de Wilks, que mostra a significância da função discriminante. Verifica-se que esta função é altamente significativa.
Com base na Tabela 3, a função discriminante pode também ser representada pela Equação (1), a seguir. Através desta equação é possível calcular os escores $(Z)$ das cidades e posicio ná-las no grupo 1 ou no grupo 2 :

$\mathrm{Z}=-5,00999+0,07816 \mathrm{X}_{1}+$

$+0,03858 \mathrm{X}_{2}-0,4122358 \mathrm{X}_{3}+$

$+0,71258 \mathrm{X}_{4}+0,000156 \mathrm{X}_{5}$

$+0,041155 \mathrm{X}_{6}$

A seguir verifica-se o ajuste da função discriminante em relação aos dados, ou seja, será calculado o escore para cada cidade e verificado se o mesmo pode alocar adequadamente a cidade em um dos dois grupos previamente definidos. Além disto, será avaliada a capacidade do modelo em classificar cidades fora da amostra.

\section{- Cálculo dos escores discriminantes}

Com a fórmula da função discriminante dada pela Equação 1 foi possível calcular o escore $\mathrm{Z}$ para cada 
Tabela 3 - Definição da função discriminante não padronizada

\begin{tabular}{ccc}
\hline & Variáveis & Coeficientes da função discriminante \\
\hline $\mathrm{X}_{1}$ & Área de recepção & 0,07815 \\
$\mathrm{X}_{2}$ & Domicílios com água & 0,03858 \\
$\mathrm{X}_{3}$ & Incentivos & $-0,41223$ \\
$\mathrm{X}_{4}$ & Programa de coleta & 0,71258 \\
$\mathrm{X}_{5}$ & Renda média & 0,000156 \\
$\mathrm{X}_{6}$ & Funcionários médios & 0,041155 \\
& Constante & $-5,00999$ \\
\hline
\end{tabular}

Nota: $\mathrm{O}$ teste de Wilks para verificar a significância da função discriminante foi de 0,640 $(\mathrm{p}$-valor $=0,004)$

observação. Observações com escores próximos supostamente pertencem ao mesmo grupo e observações com escores muito distantes provavelmente pertencem a grupos diferentes. Este procedimento de determinar a porcentagem de classificações corretas é semelhante ao conceito de coeficiente de determinação, R2, na regressão linear.

$\mathrm{Na}$ Tabela 4 encontra-se o escore da função discriminante para cada cidade da amostra em função das seis variáveis selecionadas pelo modelo. $\mathrm{O}$ escore permitiu a classificação da cidade em um grupo ou em outro. Além disso, dentro de cada grupo foi possível verificar o ranking da cidade através desse mesmo valor. De forma geral, verificou-se que o modelo permitiu classificar corretamente $85,4 \%$ das cidades da própria amostra e $77,1 \%$ fora da amostra (via opção cross-validation do SPSS), o que é considerado muito bom se comparado com outros modelos que utilizam análise discriminante, como por exemplo, modelos de previsão de insolvência, cujo grau de precisão varia de 70 a $90 \%$, conforme Sanvicente e Minardi (1998).

\section{- Avaliando as diferenças entre os escores discriminantes para os grupos}

Uma maneira de verificar a diferença entre grupos é a comparação dos centróides de cada grupo. Centróide de um grupo é a média aritmética dos escores $Z$ de todos os membros desse grupo. Uma medida do sucesso da análise discriminante é sua capacidade de definir uma função discriminante que resulte na determinação de centróides de grupos significativamente diferentes. O cálculo dos centróides auxilia o posi- cionamento da cidade num ou noutro grupo. O valor dos centróides para cada grupo é apresentado na Tabela 5.

Uma vez calculado o valor dos dois centróides, determina-se o ponto de corte, ZC, que é dado pela média ponderada dos centróides de cada grupo. A ponderação adotada neste trabalho foi em função do tamanho de cada grupo. Esse ponto de corte serve para separar os valores da função discriminante nos respectivos grupos e permite também posicionar novas cidades.

Graficamente, esta regra de classificação para dois grupos pode ser vista na Figura 1, onde é possível visualizar a posição dos centróides e do ponto de corte sob as curvas de distribuição normal, auxiliando na classificaçã̃o entre os dois grupos, que são de diferentes tamanhos.

Com base no valor dos centróides $\left(Z_{1}=-0,380\right.$ e $\left.Z_{2}=1,273\right)$, o ponto de corte $\left(Z_{C}\right)$ foi calculado da seguinte maneira: $Z_{c}=\frac{36 \times 1,273+12 \times(-0,380)}{48}=$ $=0,8598$

Para grupos de diferentes tamanhos, como é o presente caso, a regra de classificação deverá ser ponderada para que as probabilidades de classificaçôes incorretas sejam iguais para ambos os grupos, de acordo com Hair et al (1998).

\section{•Interpretação dos resultados}

Uma vez verificada a significância da função discriminante e de seu ajuste aos dados, o próximo passo foi a interpretação dos resultados. Esta etapa, de acordo com Hair et al. (1998, p. 272), envolve o exame da função discriminante para determinar a importância relativa de cada variável independente na discriminação entre os grupos.
Existem vários métodos para verificar a importância relativa de cada variável. Neste trabalho adotou-se o método denominado de "pesos discriminantes" (discriminant weights) que analisa o sinal e a magnitude da função discriminante padronizada. Quando o sinal é ignorado, cada peso ou coeficiente representa a contribuição relativa da variável associada ao coeficiente para a função discriminante.

$\mathrm{Na}$ Tabela 6, analisando o valor dos coeficientes das variáveis (ignorando o sinal), nota-se que as variáveis que mais contribuíram para a discriminação entre as cidades foram "funcionários de nível médio", "domicílios com água", "incentivos" e "renda média", com pesos discriminantes de 0,$552 ; 0,521 ; 0,498$ e 0,374 , respectivamente.

No caso da variável "funcionários de nível médio", a sua importância poderia estar associada ao aspecto de gestão. Esse aspecto, segundo Reinfeld (1994), faz parte de um processo de planejamento em termos comunitários, treinamento de especialistas e políticas chave. Na verdade, as grandes mudanças e desafios relacionados às questôes ambientais exigem das municipalidades um corpo funcional com um bom nível educacional e competência para melhor administrar essas mudanças.

A variável "domicílios com água" foi a que obteve o segundo melhor coeficiente da função discriminante padronizada, conforme mostra a Tabela 6 . Este resultado é coerente com a literatura sobre desenvolvimento sustentável. $\mathrm{O}$ acesso à água tratada é fundamental para a melhoria das condições de higiene da população, sendo um indicador universal de desenvolvimento sustentável. Trata-se de um indicador importante para as características básicas de qualidade de vida da população, além de servir de acompanhamento das políticas públicas de saneamento básico e ambiental (IBGE, 2002).

A variável "incentivos", a terceira em termos de importância no modelo em apreço, representa os mecanismos utilizados pelos municípios para atrair atividade econômica, relaciona-se ao aspecto político-legal. Assim, as cidades menos desenvolvidas são as que oferecem mais incentivos, ou seja, esse seria um indicador de fraqueza econômica do município. Por essa razão, essa variável apresentou sinal negativo na função discriminante, ou seja, as cidades que têm mais programas de 
Tabela 4 - Amostra, classificação atual e pelo modelo, valor da função Z e as seis variáveis

\begin{tabular}{|c|c|c|c|c|c|c|c|c|}
\hline Cidades & $\begin{array}{c}\text { Classif. } \\
\text { original/ } \\
\text { modelo * }\end{array}$ & $\begin{array}{c}\text { Áreas } \\
\text { recepção } \\
\text { entulhos }\end{array}$ & $\begin{array}{c}\text { Renda } \\
\text { média } \\
(\mathrm{R} \$)\end{array}$ & $\begin{array}{c}\% \\
\text { funcionários } \\
\text { nível médio }\end{array}$ & Incentivos & $\begin{array}{c}\text { Progr. } \\
\text { coleta } \\
\text { seletiva }\end{array}$ & $\begin{array}{c}\% \\
\text { domicílios } \\
\text { com água }\end{array}$ & $\begin{array}{c}\text { Escore } \\
\text { função } \\
\text { Z }\end{array}$ \\
\hline Aracaju & $1 / 1$ & 0 & $5.497,92$ & 12,12 & 4 & 1 & 95,70 & $-0,8937$ \\
\hline Bauru & $1 / 1$ & 0 & $6.644,43$ & 31,14 & 4 & 0 & 98,90 & $-0,5199$ \\
\hline Belém & $1 / 1$ & 0 & $6.301,80$ & 55,28 & 4 & 1 & 73,90 & 0,1698 \\
\hline Belo Horizonte & $2 / 2$ & 23 & $10.421,83$ & 22,54 & 2 & 1 & 99,30 & 3,0637 \\
\hline Betim & $1 / 1$ & 0 & $9.901,36$ & 26,49 & 4 & 0 & 98,00 & $-0,2382$ \\
\hline Boa Vista & $1 / 1$ & 0 & $4.000,63$ & 18,56 & 2 & 0 & 97,40 & $-0,6857$ \\
\hline Brasília & $2 / 2$ & 2 & $9.844,96$ & 67,69 & 4 & 1 & 91,80 & 2,0811 \\
\hline Campina Grande & $1 / 1$ & 0 & $2.054,82$ & 31,14 & 4 & 0 & 96,60 & $-1,3246$ \\
\hline Campinas & $2 / 1$ & 0 & $7.422,66$ & 10,97 & 4 & 1 & 97,30 & $-0,5791$ \\
\hline Canoas & $1 / 1$ & 0 & $4.920,35$ & 1,84 & 0 & 1 & 96,40 & 0,2657 \\
\hline Contagem & $1 / 1$ & 0 & $3.944,26$ & 40,61 & 4 & 1 & 99,10 & 0,1696 \\
\hline Cuiabá & $1 / 1$ & 0 & $6.633,72$ & 31,14 & 1 & 0 & 92,50 & 0,4662 \\
\hline Feira de Santana & $1 / 1$ & 0 & $2.441,10$ & 13,44 & 4 & 0 & 80,20 & $-2,6268$ \\
\hline Florianópolis & $1 / 2$ & 0 & $10.156,81$ & 38,38 & 3 & 1 & 90,50 & 1,1265 \\
\hline Fortaleza & $1 / 1$ & 0 & $3.720,22$ & 59,63 & 4 & 1 & 87,20 & 0,4595 \\
\hline Guarulhos & $2 / 2$ & 5 & $6.363,39$ & 42,62 & 2 & 0 & 94,70 & 0,9614 \\
\hline Juiz de Fora & $1 / 1$ & 0 & $5.928,41$ & 40,20 & 4 & 1 & 96,00 & 0,3426 \\
\hline Londrina & $2 / 1$ & 1 & $6.242,99$ & 31,14 & 3 & 0 & 97,20 & $-0,1584$ \\
\hline Macapá & $1 / 1$ & 0 & $3.428,54$ & 46,60 & 2 & 0 & 54,30 & $-1,2820$ \\
\hline Maceió & $1 / 1$ & 0 & $3.489,98$ & 20,16 & 4 & 0 & 81,90 & $-2,1205$ \\
\hline Manaus & $1 / 1$ & 0 & $5.857,58$ & 41,93 & 4 & 0 & 74,90 & $-1,1239$ \\
\hline Maringá & $1 / 1$ & 0 & $7.338,03$ & 31,14 & 4 & 1 & 93,90 & 0,1080 \\
\hline Mauá & $1 / 1$ & 0 & $6.044,92$ & 9,94 & 2 & 0 & 98,20 & $-0,6912$ \\
\hline Mogi das Cruzes & $1 / 1$ & 0 & $8.044,63$ & 18,01 & 4 & 0 & 91,80 & $-1,1167$ \\
\hline Montes Claros & $1 / 1$ & 0 & $3.452,63$ & 33,19 & & 0 & 96,90 & 0,6358 \\
\hline Natal & $1 / 1$ & 0 & $5.218,56$ & 40,14 & 4 & 0 & 97,20 & $-0,4369$ \\
\hline Niterói & $1 / 1$ & 0 & $7.467,59$ & 32,84 & 4 & 1 & 78,30 & $-0,4037$ \\
\hline Osasco & $1 / 1$ & 0 & $5.701,94$ & 25,00 & 4 & 0 & 98,60 & $-0,9316$ \\
\hline Palmas & $1 / 1$ & 0 & $1.381,41$ & 33,22 & 4 & 0 & 94,60 & $-1,4211$ \\
\hline Pelotas & $1 / 1$ & 0 & $4.417,77$ & 54,05 & 3 & 0 & 96,80 & 0,4077 \\
\hline Petrópolis & $1 / 1$ & 0 & $5.288,02$ & 41,04 & 2 & 0 & 52,10 & $-1,3061$ \\
\hline Piracicaba & $2 / 2$ & 2 & $9.825,21$ & 37,33 & 0 & 1 & 99,40 & 2,7659 \\
\hline Porto Alegre & $1 / 2$ & 0 & $10.795,78$ & 31,14 & 3 & 1 & 98,70 & 1,2442 \\
\hline Porto Velho & $1 / 1$ & 0 & $6.778,35$ & 35,00 & 0 & 0 & 40,10 & $-0,9625$ \\
\hline Recife & $1 / 1$ & 0 & $5.504,19$ & 23,00 & 4 & 1 & 88,00 & $-0,7413$ \\
\hline
\end{tabular}


Tabela 4 - Amostra, classificação atual e pelo modelo, valor da função Z e as seis variáveis (continuação)

\begin{tabular}{ccccccccc}
\hline Cidades & $\begin{array}{c}\text { Classif. } \\
\text { original/ } \\
\text { modelo }\end{array}$ & $\begin{array}{c}\text { Áreas } \\
\text { recepção } \\
\text { entulhos }\end{array}$ & $\begin{array}{c}\text { Renda } \\
\text { média } \\
(\mathrm{R} \$)\end{array}$ & $\begin{array}{c}\% \\
\text { funcionários } \\
\text { nível médio }\end{array}$ & $\begin{array}{c}\text { Incentivos } \\
\text { Progr. }\end{array}$ & $\begin{array}{c}\text { P } \\
\text { coleta } \\
\text { seletiva }\end{array}$ & $\begin{array}{c}\text { Escore } \\
\text { domicílios } \\
\text { com água }\end{array}$ & $\begin{array}{c}\text { função } \\
Z\end{array}$ \\
\hline Ribeirão Preto & $2 / 1$ & 1 & $7.386,06$ & 30,51 & 4 & 1 & 98,00 & 0,3259 \\
Rio Branco & $1 / 1$ & 0 & $5.354,98$ & 41,59 & 4 & 0 & 55,80 & $-1,9533$ \\
Rio de Janeiro & $2 / 2$ & 0 & $8.679,28$ & 31,14 & 1 & 1 & 97,80 & 1,7024 \\
S.B. do Campo & $1 / 1$ & 0 & $10.372,43$ & 31,14 & 4 & 1 & 98,00 & 0,7395 \\
S.J dos Campos & $2 / 1$ & 1 & $11.912,95$ & 26,54 & 4 & 1 & 96,10 & 0,7951 \\
S.J. do Rio Preto & $2 / 2$ & 6 & $5.960,86$ & 56,80 & 4 & 1 & 96,60 & 1,5241 \\
Salvador & $2 / 2$ & 6 & $5.675,27$ & 48,28 & 4 & 1 & 96,60 & 1,1283 \\
Santo André & $1 / 2$ & 15 & $9.786,93$ & 23,83 & 4 & 1 & 97,00 & 1,4807 \\
Santos & $1 / 1$ & 0 & $10.546,29$ & 18,11 & 4 & 1 & 99,70 & 0,2951 \\
São Paulo & $2 / 2$ & 6 & $10.313,03$ & 31,14 & 3 & 1 & 99,40 & 1,6648 \\
Sorocaba & $1 / 1$ & 0 & $6.779,61$ & 27,42 & 3 & 0 & 99,30 & $-0,2251$ \\
Teresina & $1 / 1$ & 0 & $3.281,78$ & 30,05 & 4 & 0 & 93,70 & $-1,2900$ \\
Uberlândia & $1 / 1$ & 19 & 4899,7 & 31,14 & 4 & 0 & 99,30 & 0,7084 \\
Média Grupo 1 & & 0,94 & $5.927,15$ & 31,10 & 3,25 & 0,39 & 88,38 & $-0,3799$ \\
Média Grupo 2 & & 4,42 & $8.337,37$ & 36,39 & 2,92 & 0,83 & 97,02 & 1,2729 \\
\hline
\end{tabular}

Nota:* Na segunda coluna da tabela, o primeiro número refere-se à classificação original da cidade e o segundo à classificação

Pelo modelo.

Tabela 5 - Valor médio da função discriminante para os centróides de cada grupo

\begin{tabular}{cc}
\hline Grupo & Valor médio da função \\
\hline 1 & $Z_{1}=-0,380$ \\
2 & $Z_{2}=1,273$ \\
\hline
\end{tabular}

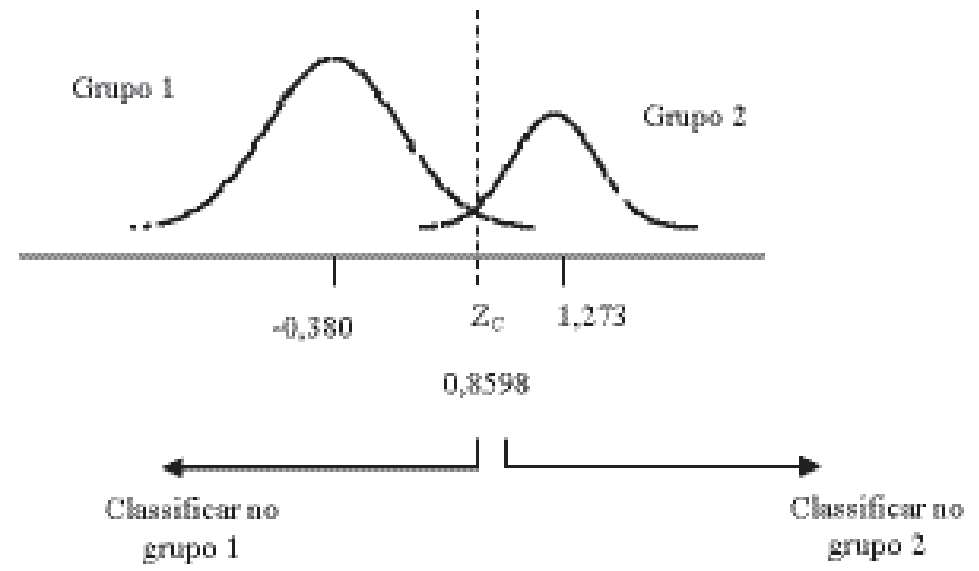

Fonte: Adaptado de Hair et al (1998)

Figura I - Representação dos centróides e do ponto de corte 
Tabela 6 - Coeficientes da função discriminante padronizada

\begin{tabular}{cc}
\hline Variável & Coeficientes da função discriminante \\
\hline Área de recepção & 0,363 \\
Domicílios com água & 0,521 \\
Incentivos & $-0,498$ \\
Programas de coleta & 0,336 \\
Renda média & 0,374 \\
Funcionário médio & 0,552 \\
\hline
\end{tabular}

incentivos obtiveram um escore menor, pois, supostamente, devem ser menos desenvolvidas economicamente.

A variável "renda média" é considerada por Languell (2001) como um indicador da saúde econômica da região e da população, impulsionando a atividade econômica e, por conseguinte, a atividade construtiva, que gera mais resíduo. Esse é um fator a contribuir para a reciclagem, pois, por um lado é necessário que haja matériaprima suficiente para suprir as usinas de reciclagem e, por outro lado, mostra que há novas construções e obras de infra-estrutura que podem absorver o material reciclado. Ou seja, há oferta e demanda para alimentar esse processo, o que só acontece em situaçôes de maior desenvolvimento econômico.

A variável "área de recepção" (de entulhos) pode ser considerada uma condição para facilitar a implantação de um programa de reciclagem. Uma grande quantidade de áreas de recepção indica que existe uma rede organizada e estruturada, que serve como centro de triagem, diminuindo assim os custos de transporte e promovendo a reciclagem.

Finalmente, a sexta variável mais importante, segundo o modelo estimado, foi "programas de coleta seletiva". Esta variável está relacionada à gestão municipal e a aspectos técnicos da reciclagem.

Portanto, observa-se que das seis variáveis mais importantes quatro estão relacionadas aos aspectos de gestão municipal e técnicos de reciclagem (área de recepção, domicílios com água, programas de coleta seletiva e funcionários de nível médio), uma variável se relaciona ao aspecto econômico (renda média) e a última se relaciona a fatores político-legais (incentivos). Observa-se que a variável "domicílios com água” também está relacionada aos fatores sociais e econômicos, pois é um indicador universal de desenvolvimento sustentável.

\section{-Aplicação do modelo na classificação de alguns municípios}

Analisando as informações da Tabela 4 observa-se que as cidades que ocupam as primeiras posições foram Belo Horizonte, Piracicaba e Brasília. As cidades com as piores posiçôes, de acordo com o escore obtido da função discriminante, foram: Rio Branco, Maceió e Feira de Santana. Será que estas classificaçõos dadas pela função discriminante estão corretas? Correspondem à realidade de cada cidade?

Para responder a essas perguntas descrever-se-á a situação de algumas destas cidades em relação à sua postura quanto aos programas de reciclagem de RCD para verificar se corresponde à classificação obtida pela função discriminante.

Belo Horizonte: de acordo com a Tabela 4, Belo Horizonte foi a cidade melhor posicionada no grupo 2 (com reciclagem). Este fato corresponde à realidade, pois conforme Paulics (2000), Belo Horizonte é considerada referência em âmbito nacional em termos de implantação de reciclagem de RCD, sendo das primeiras cidades a implantar programa de reciclagem de RCD com sucesso. $\mathrm{O}$ escore alcançado pela cidade é influenciado pela variável "domicílio com água” (99,3\%), o maior número de "áreas de recepção de entulhos" (23 áreas), e "renda média anual" de $\mathrm{R}$ \$ $10.421,83$, todos esses valores são maiores que a média das variáveis que compóem o grupo 2.

Piracicaba: ocupou a segunda posição na Tabela 4, pois, como Belo Horizonte, tem um desempenho superior à média do grupo 2 no que se refere às variáveis "renda média" (R\$ 9.825,21), "domicílios com água” (99,4\%), "funcionários de nível mé- dio" (37,33\%), "programas de coleta seletiva" e "incentivos". Notar que para essa última variável, quanto menos incentivos, melhor! Somente em relação à variável "áreas de recepção" (2 áreas), seu valor está abaixo da média do grupo 2. Segundo informações da prefeitura, a cidade é uma das únicas no Brasil a ter implantado seu planejamento estratégico com base nos princípios da Agenda 21, através do projeto Piracicaba 2010 (transformado em ONG), com vistas a seu desenvolvimento sustentável.

Brasília: cidade que possui programa de reciclagem, ocupando a terceira posição geral. Seu alto escore foi obtido, principalmente, pelo alto "percentual de funcionários de nível médio" (76,69\%), quase o dobro do valor médio alcançado pela amostra do grupo 2. Somente na variável "domicílio com água” é que seu valor fica abaixo da média do grupo 2. Esta cidade não foi mais bem posicionada por causa do alto valor da variável "incentivo" que, por ter um sinal negativo, contribuiu para a redução do escore da função discriminante.

Feira de Santana: com relação às cidades que constituem o grupo 1 (sem reciclagem), Feira de Santana obteve o pior posicionamento. Além de não possuir programa de coleta seletiva, sua renda média está entre as mais baixas. $\mathrm{Na}$ verdade, nos serviços de saneamento básico seu valor está abaixo da média do grupo 1, a porcentagem de domicílios com serviço de água é de 80,20\%, abaixo da média do grupo 1 . Além disso, a porcentagem de funcionários da administração municipal de nível médio é de $13,44 \%$, quase a metade do valor da média do grupo 1 .

Maceió: é a segunda cidade pior posicionada, pois além de não possuir área de recepção e programa de coleta seletiva, possui um desempenho menor que a média do grupo $1 \mathrm{em}$ todas as variáveis da função discriminante. A variável "incentivos" apresenta-se alta, caracterizando municípios pouco desenvolvidos, como mencionado anteriormente.

Rio Branco: é a terceira cidade pior posicionada. Observa-se que, além de não possuir área de recepção e programa de coleta seletiva, a variável "domicílios com água” (55,80\%) apresenta valor muito abaixo da média do grupo 1. Segundo as variáveis "incentivo" e "renda média", a cidade apresenta um posicionamento quase igual à média do grupo 1 . 
Enfim, é possível perceber que a análise discriminante é um ferramental útil que possibilita realizar uma análise minuciosa quando se trata de um grande número de observaçóes e variáveis inter-relacionadas. Os exemplos vistos acima mostraram que o escore obtido por cada cidade, utilizando-se apenas seis das 20 variáveis iniciais, foi coerente com a realidade de cada uma.

\section{- Classificando cidades fora da amostra}

A seguir, analisa-se a capacidade de classificação do modelo para cidades fora da amostra. O objetivo desta etapa é o de verificar a coerência dos resultados do modelo no posicionamento de uma nova cidade. Para tanto, apresentase a Tabela 7 que contém os dados para algumas cidades fora da amostra. Estas foram as cidades retiradas do grupo 1 pelo procedimento aleatório, descrito na seção 3 .

Observa-se, na Tabela 7, que o modelo classificou corretamente 14 das 17 cidades apresentadas (82,35\%). Este valor não pode ser comparado ao procedimento interno de cross-validation do programa SPSS, pois se refere apenas às cidades do grupo 1 . Naquele procedimento, a média de classificaçôes corretas para as cidades dos grupos $1 \mathrm{e}$ 2 foi de $77,1 \%$.

Além da possibilidade de classificar uma nova cidade, o modelo pode ser usado como ferramenta para verificar se determinada cidade possui ou não as condições favoráveis para o sucesso da implantação de um programa de reciclagem de RCD.

Exemplificando, verifica-se na Tabela 7, que a cidade de Jundiaí apresenta um escore para a função discriminante de $Z=2,0511$, um valor maior que o ponto de corte $\left(Z_{C}=0,8598\right)$. Este valor posiciona Jundiaí no grupo 2, apesar de que na classificação original estar situada no grupo 1 (sem reciclagem de RCD). A classificação fornecida pelo modelo é coerente, pois a cidade apresenta valores para a maioria das variáveis da Equação 1 superiores à média do grupo 2 . $\mathrm{O}$ único fator negativo é não possuir área de recepção de resíduos. Assim, pelos dados apresentados, é possível inferir que essa cidade apresenta condições propícias para que um programa de reciclagem se desenvolva com sucesso.
Por outro lado, verifica-se que a cidade de São Luís obteve um escore de $Z=-1,675$, um valor menor que o ponto de corte $\left(Z_{C}\right)$. Assim, esta cidade posiciona-se no grupo 1 , estando de acordo com a situação atual. Este baixo escore justifica-se principalmente por ela não ter área de recepção e programa de coleta seletiva, além do fato dos valores apresentados pelas variáveis "renda média” e "domicílios com água” estarem abaixo da média do grupo 1 . Somente com relação à variável "funcionários de nível médio" $(31,14 \%)$ seu valor foi igual à média do grupo 1 . Pelos dados apresentados, essa cidade demonstra uma situação precária. Verifica-se, assim, que esse município deve apresentar alguns obstáculos à implantação de um programa de reciclagem.

Salienta-se, contudo, que o modelo apresentado não deve ser aplicado cegamente, pois é apenas um modelo para subsidiar o planejamento de programas de RCD.

\section{CONCLUSÕES}

O presente trabalho teve como objetivo desenvolver um modelo

Tabela 7 - Classificação de cidades fora da amostra

\begin{tabular}{ccccccccc}
\hline Cidades & $\begin{array}{c}\text { Classif. } \\
\text { Original/ } \\
\text { Modelo* }\end{array}$ & $\begin{array}{c}\text { Áreas } \\
\text { recepção } \\
\text { entulhos }\end{array}$ & $\begin{array}{c}\text { Renda } \\
\text { média } \\
(\mathrm{R} \$)\end{array}$ & $\begin{array}{c}\text { \% } \\
\text { funcionários } \\
\text { nível médio }\end{array}$ & $\begin{array}{c}\text { Incentivos } \\
\text { Programa } \\
\text { de coleta } \\
\text { seletiva }\end{array}$ & $\begin{array}{c}\% \\
\text { domić́lios } \\
\text { com água }\end{array}$ & $\begin{array}{c}\text { Escore } \\
\text { função } \\
\text { Z }\end{array}$ \\
\hline Belford Roxo & $1 / 1$ & 0 & $2.237,51$ & 31,14 & 3 & 0 & 72,20 & $-1,8260$ \\
Boa Vista & $1 / 1$ & 0 & $4.000,63$ & 18,56 & 2 & 0 & 97,40 & $-0,6857$ \\
Campo Grande & $1 / 1$ & 0 & $6.493,64$ & 29,71 & 4 & 0 & 88,80 & $-0,9921$ \\
Campos dos & $1 / 1$ & 0 & $3.570,56$ & 19,42 & 4 & 0 & 73,90 & $-2,4472$ \\
Goytacazes & & & & & & & & \\
Cariacica & $1 / 1$ & 0 & $2.448,49$ & 31,14 & 4 & 0 & 96,40 & $-1,2709$ \\
Caxias do Sul & $1 / 2$ & 0 & $6.339,25$ & 31,14 & 2 & 1 & 97,70 & 0,9219 \\
Curitiba & $1 / 1$ & 0 & $9.067,90$ & 10,81 & 3 & 1 & 98,60 & 0,1327 \\
Diadema & $1 / 1$ & 2 & $6.872,69$ & 31,14 & 4 & 1 & 99,10 & 0,3924 \\
Goiânia & $1 / 1$ & 0 & $5.919,38$ & 19,08 & 4 & 0 & 87,90 & $-1,5546$ \\
João Pessoa & $1 / 1$ & 0 & $4.200,84$ & 27,36 & 4 & 0 & 97,70 & $-1,1032$ \\
Joinville & $1 / 1$ & 0 & $5.342,70$ & 30,34 & 4 & 0 & 96,40 & $-0,8524$ \\
Jundiaí & $1 / 2$ & 0 & $10.917,63$ & 41,58 & 2 & 1 & 97,30 & 2,0511 \\
Olinda & $1 / 1$ & 0 & $2.774,73$ & 23,55 & 1 & 0 & 94,40 & $-0,3754$ \\
São Luis & $1 / 1$ & 0 & $3.818,02$ & 31,14 & 4 & 0 & 80,40 & $-1,6746$ \\
São Vicente & $1 / 1$ & 0 & $3.290,28$ & 13,69 & 3 & 1 & 99,40 & $-0,6190$ \\
Vila Velha & $1 / 1$ & 0 & $2.319,38$ & 30,42 & 2 & 0 & 98,40 & $-0,4204$ \\
Vitória & $1 / 2$ & 0 & $10.879,08$ & 34,95 & 4 & 1 & 99,30 & 1,0258 \\
\hline
\end{tabular}

Notas: * O primeiro número da coluna significa a classificação original da cidade e o segundo a classificação obtida pelo modelo. 
quantitativo, baseado em técnicas exploratórias de dados e de estatística multivariada, que permite identificar e caracterizar as variáveis mais relevantes, a nível social, econômico, político-legal e técnico-gerenciais associadas ao sucesso da implantação de programas de reciclagem de RCD nos municípios brasileiros.

A aplicação da análise discriminante permitiu identificar, entre as 20 variáveis iniciais, as seis que mais contribuíram para a discriminação entre as cidades que possuem e não possuem programas de reciclagem. A função discriminante estimada permitiu classificar corretamente $85,4 \%$ das cidades da própria amostra e $77,1 \%$ fora $\mathrm{da}$ amostra, o que é considerado muito bom se comparado a outros modelos que utilizam essa técnica.

Importante observar que foi utilizada a técnica de regressão logística como alternativa à análise discriminante. Como os resultados foram semelhantes, apresentados na Tabela 8, optou-se pelo uso da análise discriminante por ser esta capaz de trabalhar com menor relação observaçôes/variáveis independentes, conforme Hair et al (1998, p. 258), e também pelo software utilizado dispor da opção para se efetuar um teste de cross-validation tipo leave-one-out, que verifica o grau de acerto do modelo em prever uma classificação correta para cidades fora da amostra. Isto é útil quando a amostra é pequena e não se pode dividi-la em duas partes, uma para estimação e outra para validação.

O método de análise revelou que as cidades mais propensas à reciclagem ou as que receberam o escore mais alto, são aquelas que apresentaram melhor desempenho principalmente nas variáveis relacionadas à gestão municipal e aspectos técnicos da reciclagem. Por outro lado, as cidades que obtiveram um escore baixo, apresentaram condiçōes precárias nos seus sistemas de saneamento básico, educação e nos aspectos de gestão. Infere-se que estas cidades terão dificuldades na implementação de programas de reciclagem, visto que devem resolver outros problemas básicos, pois carecem de infra-estrutura para apoiar a reciclagem de RCD.

Interessante notar que das seis variáveis selecionadas, a maioria relacionase diretamente ou indiretamente com a gestão municipal (áreas de recepção de entulho, programas de coleta, funcionários de nível médio, domicílios com
Tabela 8 - Comparando resultados: regressão logística e análise discriminante Variáveis finais selecionadas pelo modelo

Regressão logística

Análise discriminante

Conselho

Área de recepção

Domicílios com água

Domicílios com água

Incentivos

Incentivos

Consórcio

Renda média

Programas de coleta

uncionário médio

Renda média

Funcionário médio

Classificação correta para casos dentro da amostra

$85,4 \%$

$85,4 \%$

Notas:

(1) Nos resultados da regressão logística foi utilizado o programa SPSS, com o método stepwise (backward: Wald), com tolerância de $10 \%$ para as variáveis que entram na regressão e de $20 \%$ para as que saem - mesmo procedimento aplicado na análise discriminante;

(2) Como o método iterativo, por máxima verossimilhança, usado pela regressão logística não convergia para a mesma base de dados, usou-se a variável $\log ($ densidade) na sua forma não transformada.

água), tanto se usando análise discriminante como regressão logística. Portanto, de acordo com o modelo proposto, o sucesso da implantação de programas de reciclagem de RCD parece estar nas mãos das próprias prefeituras.

Finalmente, espera-se que o uso deste tipo de metodologia possa contribuir para um melhor planejamento de estratégias que facilitem a implantação de programas de reciclagem de RCD, tornando essa implantação mais transparente, menos onerosa e com maiores chances de sucesso.

\section{REFERÊNCIAS}

ANGULO, S. C. et al. Desenvolvimento de novos mercados para a reciclagem massiva de $R C D$. In: V SEMINÁRIO DE DESENVOLVIMENTO SUSTENTÁVEL E A RECICLAGEM NA CONSTRUÇÃO CIVIL. CT - 206 IBRACON, São Paulo, 12f. 2002.

BARTH, H. P. Financial, economical and political aspects of the reuse of construction and demolition waste. In: LAURITZEN, E. K. (Org.) Demolition and reuse of concrete and masonry: Proceedings of the Third International RILEM Symposium. London: Spon Press, 552p. 1994.

CHUNG, S. S.; LO, C. W. H. Evaluating sustainability in waste management: the case of construction and demolition, chemical and clinical wastes in Hong Kong. Resources, Conservation and Recycling, v. 37 (2), p. 119-145, 2003.

FRANKLIN ASSOCIATES. Characterization of building-related construction and demolition debris in the United States. A report to the U.S. Environmental Protection Agency, Office of Solid Waste and Energy Response, Washington, D.C., $94 f .1998$.

HAIR, J. F. et al. Multivariate Data Analysis. $5^{\text {th }}$ ed., New Jersey: Prentice Hall, 730p. 1998.
IBGE. Indicadores de desenvolvimento sustentável: Brasil 2002. Rio de Janeiro: IBGE, 191f. 2002.

ICF INCORPORATED. Construction and demolition waste landfills. Prepared for U.S. Environmental Protection Agency, Office of Solid Waste, Draft Report, 39f. 1995.

JOHNSON, R. A.; WICHERN, D. W. Applied multivariate statistical analysis. New Jersey: Prentice Hall, 816p. 1998.

KLECKA, W. R. Discriminant Analysis. London: Sage Publications, Sage University Papers no 19, 71p. 1980.

LANGUELL, J. L. Development of a prototype assessment tool to evaluate the potencial to successfully implement deconstruction as a regional waste reduction strategy. Ph.D. thesis, Department of Coastal Engineering, University of Florida, 232f. 2001.

LAURITZEN, E. K.; HANSEN, T. Recycling of construction and demolition waste 1986 - 1995. Denmark: Ministry of Environment and Energy, 82f. 1997.

LEITE, M. B. Avaliação de propriedades mecânicas de concretos produzidos com agregados reciclados de resíduos de construçāo e demoliçāo. Tese (Doutorado em Engenharia Civil), UFRGS, 270f. 2001.

PAULICS, V.(Org). 125 dicas - Idéias para a ação municipal. São Paulo: Pólis, 287p. 2000.

PINTO, T. P. Metodologia para a gestão diferenciada de resíduos sólidos da construção urbana. Tese (Doutorado em Engenharia Civil), USP, 189f. 1999.

REINFELD, N. V. Sistemas de reciclagem comunitária: do projeto à administração. São Paulo: Makron Books, 258p. 1994.

SANVICENTE, A. Z.; MINARDI, A. Identificação de indicadores contábeis significativos para previsão de concordata de empresas. Working Paper, Ibmec, São Paulo, 12p. 1998.

SARDÁ, M. C. Diagnóstico do entulho gerado no municipio de Blumenau-SC: potencialidades de uso em obras públicas. Dissertação (Mestrado em Engenharia Civil), UFSC, 129f. 2003. 
VYNCKE, J.; ROUSSEAU, E. Recycling of construction and demolition waste in Belgium: actual situation and future evolution. In: LAURITZEN, E. K. (Org.) Demolition and reuse of concrete and masonry: Proceedings of the Third International RILEM Symposium. London: Spon Press, 552p. 1994

WRIGHT, P. L.; KROLL, M.J.; PARNELL, J. A. Strategic management: concepts and cases. $4^{\text {th }}$ ed. New Jersey: Prentice Hall, 780p. 1998.

Endereço para correspondência:

Nébel da Costa

Fundação Centros de Referência em Tecnologias Inovadoras

(CERTI)

Campus da Universidade Federal de Santa Catarina

Caixa Postal 5053

88040-970 Florianópolis - SC -

Brasil

Tel.:(48) 3239-2000

Fax: (48) 3239-2009

E-mail: necosta25@hotmail.com 